\title{
Molecular Level One-Step Activation of agar to Activated Carbon for High Performance Supercapacitors
}

Lixing Zhang ${ }^{a}$, Huazhi $\mathrm{Gu}{ }^{\mathrm{a}}$, Haibo Sun ${ }^{\mathrm{a}}$, Feifei Cao ${ }^{\mathrm{d}}$, Yao Chen ${ }^{\mathrm{a}, *}$ and George Zheng Chen ${ }^{a, b, c}$

${ }^{\mathrm{a}}$ The State Key Laboratory of Refractories and Metallurgy, College of Materials and Metallurgy, Wuhan University of Science and Technology, Wuhan 430081, P. R. China.

${ }^{\mathrm{b}}$ Department of Chemical and Environmental Engineering, Faculty of Science and Engineering, University of Nottingham Ningbo China, Ningbo 315100, P. R. China

${ }^{c}$ Department of Chemical and Environmental Engineering, Faculty of Engineering, University of Nottingham, Nottingham NG2 7RD, UK

${ }^{\mathrm{d}}$ College of Science, Huazhong Agricultural University, No.1 Shizishan Street, Hongshan District, Wuhan, 430070, P. R. China

${ }^{*}$ Corresponding author.

E-mail address: y.chen@wust.edu.cn (Y. Chen). 


\begin{abstract}
Activated carbon was synthesized by a simple one-step calcination of deoxygenated agar in a hot $\mathrm{KOH}$ aqueous solution, in which $\mathrm{KOH}$ plays both deoxidant and activation agent. The deoxygenation course omits high temperature carbonization in the conventional technology and leads to molecular level activation of agar in subsequent one-step calcination. The one-step activated carbon has superior specific surface area of $1672 \mathrm{~m}^{2} \mathrm{~g}^{-1}$ and total pore volume of $0.81 \mathrm{~cm}^{3} \mathrm{~g}^{-1}$. It also shows a maximum specific capacitance of $226 \mathrm{~F} \mathrm{~g}^{-1}$ in the $\mathrm{KOH}$ electrolyte, which is 1.4 times as high as that for the activated carbon by the conventional two-step method. This study provides potentially economical and effective means for the production of commercial activated carbon with high porosity for supercapacitors.
\end{abstract}




\section{Introduction}

Because traditional fossil fuels which cause environmental pollution and global climate warming are limited, searching for green energy and new energy storage devices is imminent. Supercapacitors are one type of the most important energy storage devices, which show higher power and longer lifetime comparing with second batteries [1-3]. Intrinsic supercapacitors are based on electrochemical double layer (EDL) capacitance mechanism that store and release energy by charge separation at the interface between an electrode and an electrolyte. Various carbon materials have been used in the electrodes of EDL capacitors, including activated carbon, carbon nanotubes and graphene [4-10]. Meanwhile, transition metal oxides and conductive polymers are employed as the electrode materials for pseudocapacitors based on the other Faradic reaction mechanism. Comparing with these pseudocapacitance materials, carbon materials possess absolute advantages in high power and lifetime except relative low specific capacitance [11-13]. Additionally, pseudocapacitance materials also shows low specific capacitance in ionic liquid, for example, $\mathrm{MnO}_{2}$ has a specific capacitance of $100 \mathrm{~F} \mathrm{~g}^{-1}$ in doped Butylmethylpyrrolidinium-dicyanamide with $3 \mathrm{~V}$ of potential window [14]. Therefore, the development of carbon materials toward the desire of high specific capacitance has been arousing widespread interest in supercapacitors.

Among all carbon materials, activated carbon is the earliest commercial electrode of supercapacitors due to its porous structures with large specific surface area, good electrical conductivity, excellent physical, chemical and electrochemical 
stability and low cost [15-20]. In the conventional approach, a two-step process composed of carbonization and subsequent activation of carbon rich precursors is usually applied to prepare activated carbon. While these carbon based supercapacitors demonstrate excellent properties, the two-step technology reducing high cost per $\mathrm{kWh}$ has limited the competition of supercapacitors with lithium-ion batteries in many applications [21]. Hence it is necessary to increase the capacitance of carbon electrodes using a more simple and effective approach in the electrode processing currently used in the manufacture of supercapacitors. Up to date, only two reports on one-step calcination of the mechanical mixture of biomass or agar and $\mathrm{KOH}$ have executed activation with similar yield and properties compared with those that are prepared via the conventional two-step route $[22,23]$.

Herein, we report on a novel approach to fabricate activated carbon via one-step calcination of deoxygenated agar via hot $\mathrm{KOH}$ aqueous solution. $\mathrm{KOH}$ which is homogeneously mixed with agar in the hot solution by a soft chemical method plays both deoxidant and activation agent, which omits high temperature carbonization in the conventional technology and leads to molecular level activation of agar in subsequent one-step calcination. The deoxygenation mechanism of agar in the hot $\mathrm{KOH}$ solution before the one-step calcination is also revealed for the first time. The activated carbon by one-step method shows a specific surface area of $1672 \mathrm{~m}^{2} \mathrm{~g}^{-1}$, much higher than $1048 \mathrm{~m}^{2} \mathrm{~g}^{-1}$ for the activated carbon by conventional two-step method. As a result, the activated carbon by one-step method shows $226 \mathrm{~F} \mathrm{~g}^{-1}$ at $1 \mathrm{~A}$ $\mathrm{g}^{-1}$ in $6 \mathrm{M} \mathrm{KOH}$ aqueous solution, 1.4 times as high as the activated carbon by 
two-step method.

\section{Experimental}

\subsection{Preparation of samples}

$4.5 \mathrm{~g} \mathrm{KOH}$ and a $5 \mathrm{~g}$ agar were dissolved in distilled (DI) water in a water bath at 95 ${ }^{\circ} \mathrm{C}$. A black hydrogel was obtained when the reaction finished. After thorough drying, the dried hydrogel was conducted at $725^{\circ} \mathrm{C}$ for $1 \mathrm{~h}$ in Ar. Finally, the activated carbon was obtained by washing with $15 \% \mathrm{HCl}$, abundant water for 5 times, and dried at 80 ${ }^{\circ} \mathrm{C}$ for $12 \mathrm{~h}$, which is denoted as AC-1. For comparison, activated carbon was also prepared via a conventional two-step process composing of carbonization of $5 \mathrm{~g}$ agar at $725^{\circ} \mathrm{C}$ for $1 \mathrm{~h}$ and subsequent activation with $4.5 \mathrm{~g} \mathrm{KOH}$ at $725^{\circ} \mathrm{C}$ for $1 \mathrm{~h}$, which is named as $\mathrm{AC}-2$.

\subsection{Characterization of samples}

The X-ray photoelectron spectroscope (XPS) spectra were recorded on 250XI (ThermoFisher). The metal contents of the composite were analyzed using ICP-OES on IRIS Advantage ER/S (Thermo Elemental). The morphology and elemental content were obtained by scanning electron microscopy (SEM, FEI Nova 400 Nano SEM) with X-ray energy disperse spectrum (EDS). Transmission electron microscopy (TEM) morphology was investigated by JEOL JSM 2100F. A Fourier-transform Infrared (FTIR) spectrometer (Shimadzu Spectrum One FTIR) was used to analyze the change of functional groups of the precursor. The $\mathrm{N}_{2}$ adsorption/desorption isotherms were obtained at $77 \mathrm{~K}$ using automatic volumetric 
adsorption equipment (ASAP2020HD88).

\subsection{Electrochemical measurements}

A mixture of activated carbon, acetylene black and polyvinylidene difluoride with a weight ratio of $8: 1: 1$ was pasted on nickel substrates as a working electrode with a surface density of $0.45 \mathrm{mg} \mathrm{cm}^{-2}$. For the three-electrode tests, a platinum foil and a $\mathrm{Hg} / \mathrm{HgO}$ electrode or two platinum foils were used as counter and reference electrodes in $6 \mathrm{M} \mathrm{KOH}$ or pure 1-ethyl-3-methyl-imidazolium tetrafluoroborate $\left(\mathrm{EMIMBF}_{4}\right)$ as electrolytes. For the full cell tests, the activated carbon mixtures on the nickel substrates were assembled to coin-type symmetrical SCs in ambient or an $\mathrm{Ar}$ glove box (MIKROUNA) for electrolytes of $6 \mathrm{M} \mathrm{KOH}$ or pure 1-ethyl-3-methyl-imidazolium tetrafluoroborate $\left(\mathrm{EMIMBF}_{4}\right)$. Cyclic voltammetry (CV), galvanostatic charge/discharge (GCD) and electrochemical impedances spectroscopy (EIS) were measured by a CHI 660E electrochemical workstation and the cyclic performances were measured by a LAND CT2001A system.

\section{Results and discussion}

Activated carbons are usually derived from organic precursors by carbonization at high temperature with subsequent selective oxidation with $\mathrm{KOH}$ to increase the SSA and pore volume in inert atmosphere. Intrinsically, the carbonization at high temperature is a deoxygenation course of the organic precursors in order to prevent from burning out of them in subsequent $\mathrm{KOH}$ activation. Agar, consisting of agarose and agaropectin, is an organics with a $\mathrm{C} / \mathrm{O}$ atomic ratio of 1.34 . When agar was mixed 
with hot alkaline aqueous solution, it is found that the aqueous solution turned dark after a few minutes. In order to verify the reaction between agar and $\mathrm{KOH}$, the resulted dark agar gel, named D-agar, in the hot alkaline aqueous solution was separated by dialysis to remove extra $\mathrm{KOH}$. The ICP-AES analysis of D-agar reveals that the content of $\mathrm{K}$ is $2.61 \mathrm{wt} \%$, indicative of no $\mathrm{O}-\mathrm{K}$ bond in $\mathrm{D}$-agar. The EDS spectra of D-agar reveal that an average $\mathrm{C} / \mathrm{O}$ atomic ratio of $3.45 \pm 0.70$, which is much higher than 1.34 for agar. The increased $\mathrm{C} / \mathrm{O}$ atomic ratio indicates that the agar was deoxidized in hot $\mathrm{KOH}$ aqueous solution. The FTIR spectra of agar and D-agar in Fig. 1 reveal the molecular structural change of agar in the reaction. Both spectra of agar and D-agar show two small adsorption peaks at 1654 and $2890 \mathrm{~cm}^{-1}$, which are ascribed to carbonyl $(\mathrm{C}=\mathrm{O})$ from agaropectin in agar and $\mathrm{C}-\mathrm{H}$ stretching and bending vibrations. These bonds can not readily react with $\mathrm{KOH}$. The decreased intensities of the broad absorption band at $3300 \sim 3600 \mathrm{~cm}^{-1}$ and the peak at $1075 \mathrm{~cm}^{-1}$ are obviously observed for D-agar, suggesting that the hydrogen bonded hydroxyl groups and cyclic ether linkage of six membered rings in agar are removed by a dehydration reaction with $\mathrm{KOH}$ in the hot solution $[24,25]$. The elimination of oxygen functional groups can replace the carbonization step in the conventional two-step method and ensure a yield of $2.7 \%$ for activated carbon with a tapping density of $0.18 \mathrm{~g} \mathrm{~cm}^{-3}$. However, the yield for one-step derived AC-1 is still much lower than $16.2 \%$ for AC-2 with a tapping density of $0.46 \mathrm{~g} \mathrm{~cm}^{-3}$. Further, the average carbon and oxygen contents of AC-1 are determined to be 91.21 and 8.79 at $\%$ by EDS. The XPS spectrum of AC-1 shows a small peak at $286.1 \mathrm{eV}$, corresponding to $\mathrm{C}-\mathrm{O}$ bond (Fig. 
S1), while its FTIR spectrum exhibits a characteristic $\mathrm{C}-\mathrm{O}$ bond at $1220 \mathrm{~cm}^{-1}$ (Fig. $\mathrm{S} 2$ ), suggesting that oxygen in $\mathrm{AC}-1$ is in the form of $\mathrm{C}-\mathrm{O}$ bond.

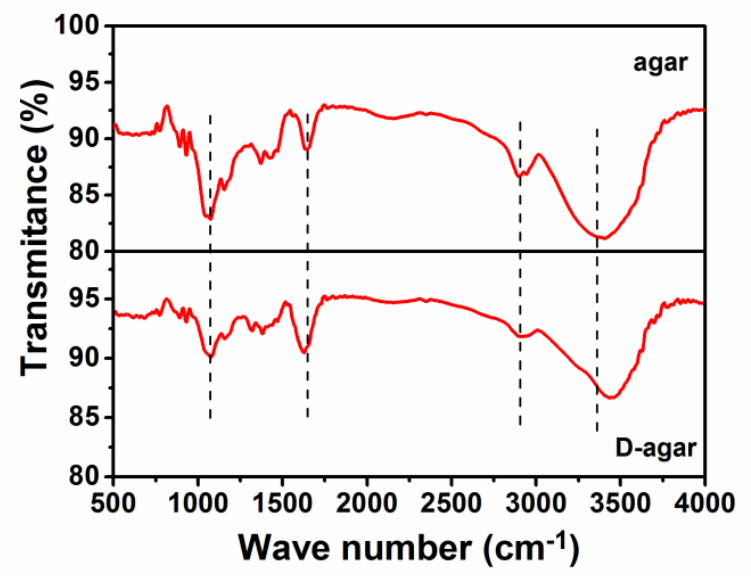

Fig. 1 FTIR spectra of agar and D-agar.

The morphologies of AC-1 and AC-2 were observed by SEM and TEM. The SEM image of $\mathrm{AC}-1$ in low and high magnification (Fig. 2a and b) show that an interconnected 3D network with macrovoids of several micrometers and their wall of a few hundreds of nanometers. The TEM image of AC-1 in Fig. 2d shows the dense micropores which are less than $1 \mathrm{~nm}$. AC-2 presents bulk characteristic with size of above $30 \mu \mathrm{m}$ composed of fractured macrovoids of tens of micrometers in Fig. 2c, which is one order of magnitude higher than that of AC- 1 . The retainence of the interconnected structure with smaller size for AC-1 are obviously attributed to a mild deoxygenation of agar instead of carbonization at high temperature. 

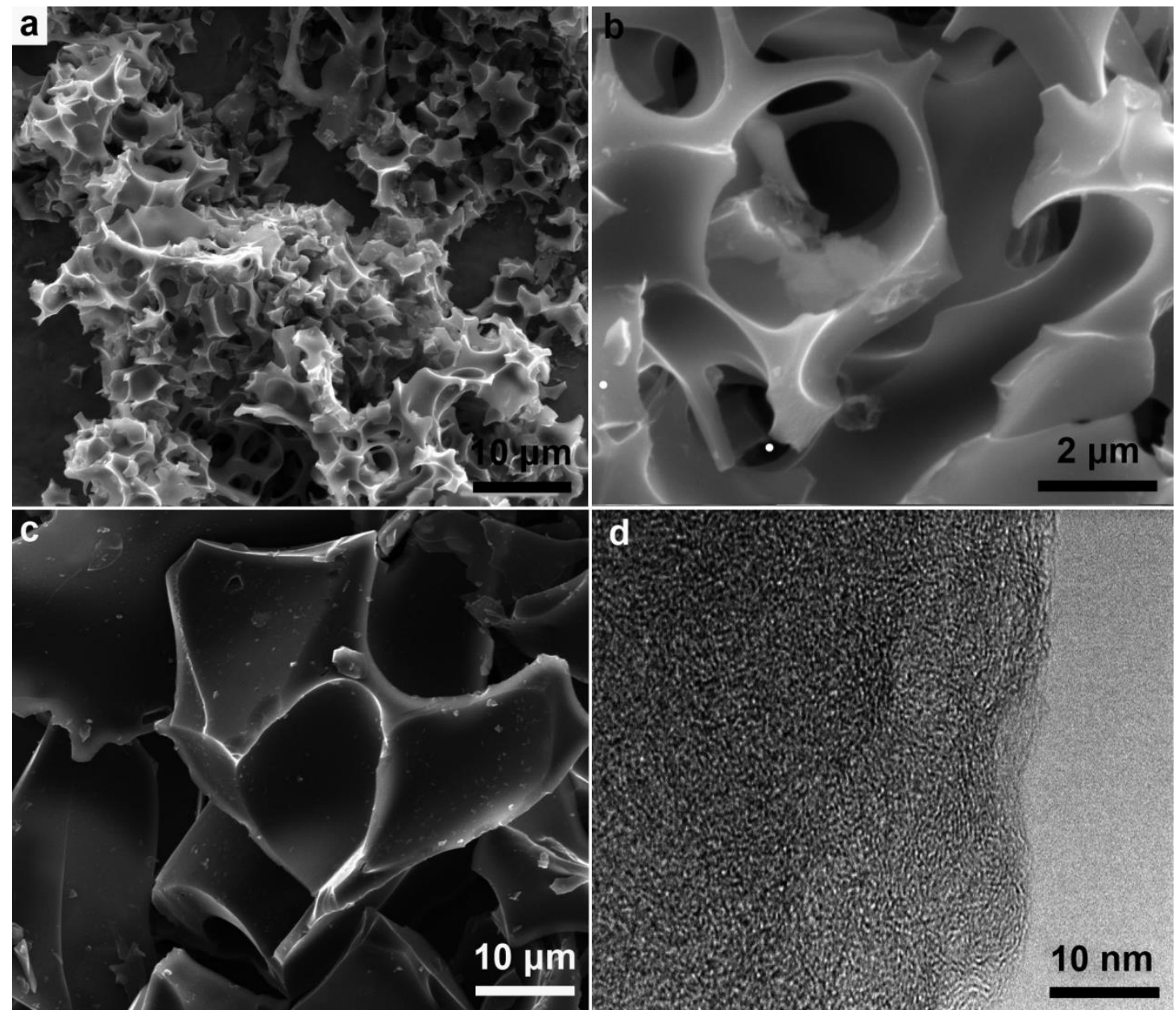

Fig. 2 (a) Low and (b) High magnification SEM images of AC-1. (c) Low magnification SEM image of AC-2. (d) High resolution TEM image of AC-1.

The adsorption and desorption isotherms of $\mathrm{N}_{2}$ at $77 \mathrm{~K}$ and the pore size distribution of AC-1 and AC-2 are shown in Fig. 3. Obviously, all the samples exhibiting a type I nitrogen adsorption desorption isotherms in the Brunauer classification [26] confirms their microporous structure (Fig. 3a), well consistent with the TEM observations. Although AC-1 and AC-2 have almost similar pore size distributions mainly ranging from 0.5 to $3 \mathrm{~nm}$ (Fig. 3b), AC-1 possesses a larger specific surface area of $1672 \mathrm{~m}^{2} \mathrm{~g}^{-1}$ and total pore volume of $0.81 \mathrm{~cm}^{3} \mathrm{~g}^{-1}$ than 1048 
$\mathrm{m}^{2} \mathrm{~g}^{-1}$ and $0.47 \mathrm{~cm}^{3} \mathrm{~g}^{-1}$ for AC-2, respectively.
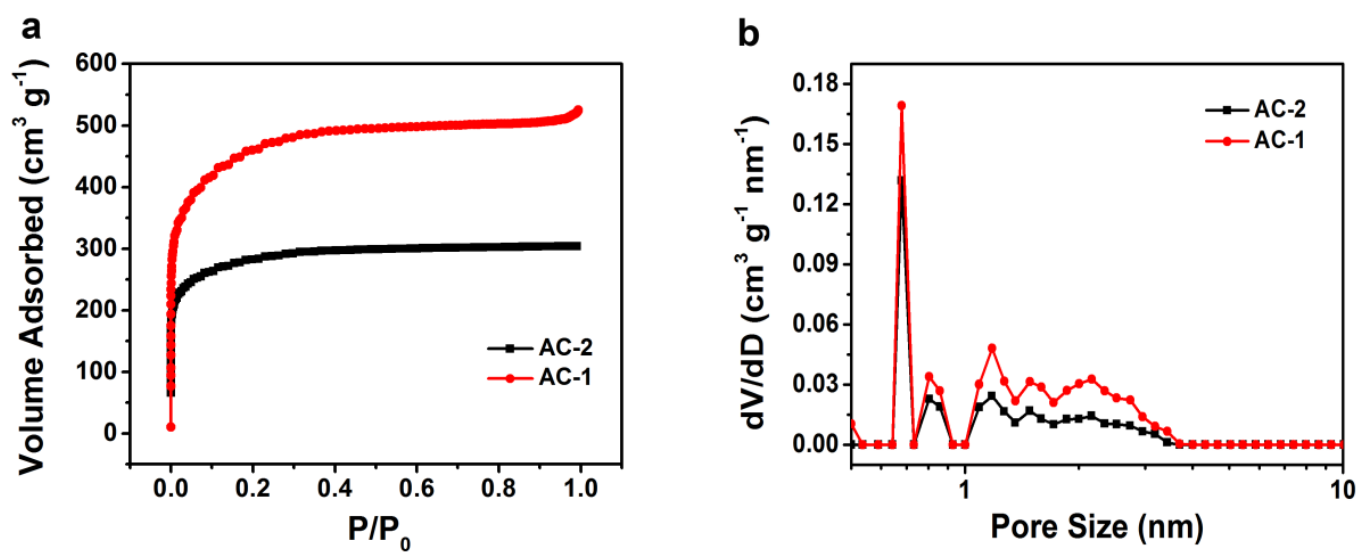

Fig. 3 (a) nitrogen adsorption-desorption isotherms and (b) pore size distribution plot of $\mathrm{AC}-1$ and $\mathrm{AC}-2$.

The electrochemical properties of $\mathrm{AC}-1$ and $\mathrm{AC}-2$ were estimated by cyclic voltammetry $(\mathrm{CV})$ and galvanostatic charge/discharge (GCD) measurements using a three-electrode cell in the potential range from 0 to $-1 \mathrm{~V}$ in $6 \mathrm{M} \mathrm{KOH}$ eletrolyte. Fig. 4a features their $\mathrm{CV}$ curves at a scan rate of $10 \mathrm{mV} \mathrm{s}^{-1}$. These $\mathrm{CV}$ profiles are quasi-rectangular in shape without obvious redox peaks, indicating that both samples have ideal capacitive behavior. However, the larger area of CV curves for the AC-1 electrode means higher specific capacitance than that of the AC-2 electrodes due to its higher specific surface area and well-developed porous structure which accommodate more electrolyte ions. Fig. 4b exhibits the GCD curves of AC-1 and AC-2. As revealed by Fig. S3, the discharge curve in a three-electrode cell within the whole potential window includes a discharge curve within relative positive potential window and a charge curve within relative negative potential window, so it is possible that the charge time is less than the discharge time in a three-electrode cell. Considering that 
columbic efficiency should not be beyond $100 \%$, the specific capacitance of AC-1 is calculated from the charging curves to be $226 \mathrm{~F} \mathrm{~g}^{-1}$ at a specific current of $1 \mathrm{~A} \mathrm{~g}^{-1}$. It is $38 \%$ higher than $164 \mathrm{~F} \mathrm{~g} \mathrm{~g}^{-1}$ of $\mathrm{AC}-2$ at the same specific current. All CV profiles of AC-1 retain a rectangular shape at different sweep rates (Fig. 4c). No obvious distortion in the $\mathrm{CV}$ curves at $200 \mathrm{mV} \mathrm{s}^{-1}$ indicates an excellent rate capability due to low equivalent series resistance and the fast diffusion of electrolyte ions into the electrode. According to Fig. 4d, the capacitance of AC-1 only decreases to $208 \mathrm{~F} \mathrm{~g}^{-1}$ at $10 \mathrm{~A} \mathrm{~g}^{-1}$, having a superb capacitive retention of $92 \%$ relative to $226 \mathrm{~F} \mathrm{~g}^{-1}$ at $1 \mathrm{~A} \mathrm{~g}^{-1}$.

Compared with AC-2, the electrochemical performances of AC-1 are evidently improved. The effective execution of the one-step method mainly relies on the deoxygenation course in the hot $\mathrm{KOH}$ solution which can increase the carbon yield. Alternatively, the deoxygenation course in one-step method extends the selection of precursors from rich carbon ones to ones with many oxygen functional groups. The deoxygenation course requires the solubility and reactive functional groups of the precursor. The high solubility of agar in hot water ensure the homogeneous deoxygenation itself with $\mathrm{KOH}$, which further leads to molecular level distribution of $\mathrm{KOH}$ in D-agar. Conversely, insoluble polyaniline without reactive functional groups via the one-step method always produces gray powders. The uniformed activation of agar produces interconnected 3D network with small size macrovoids, also meaning that more pores are produced in more external surface. As a result, larger capacitance is obtained in AC-1 by the one-step method. 

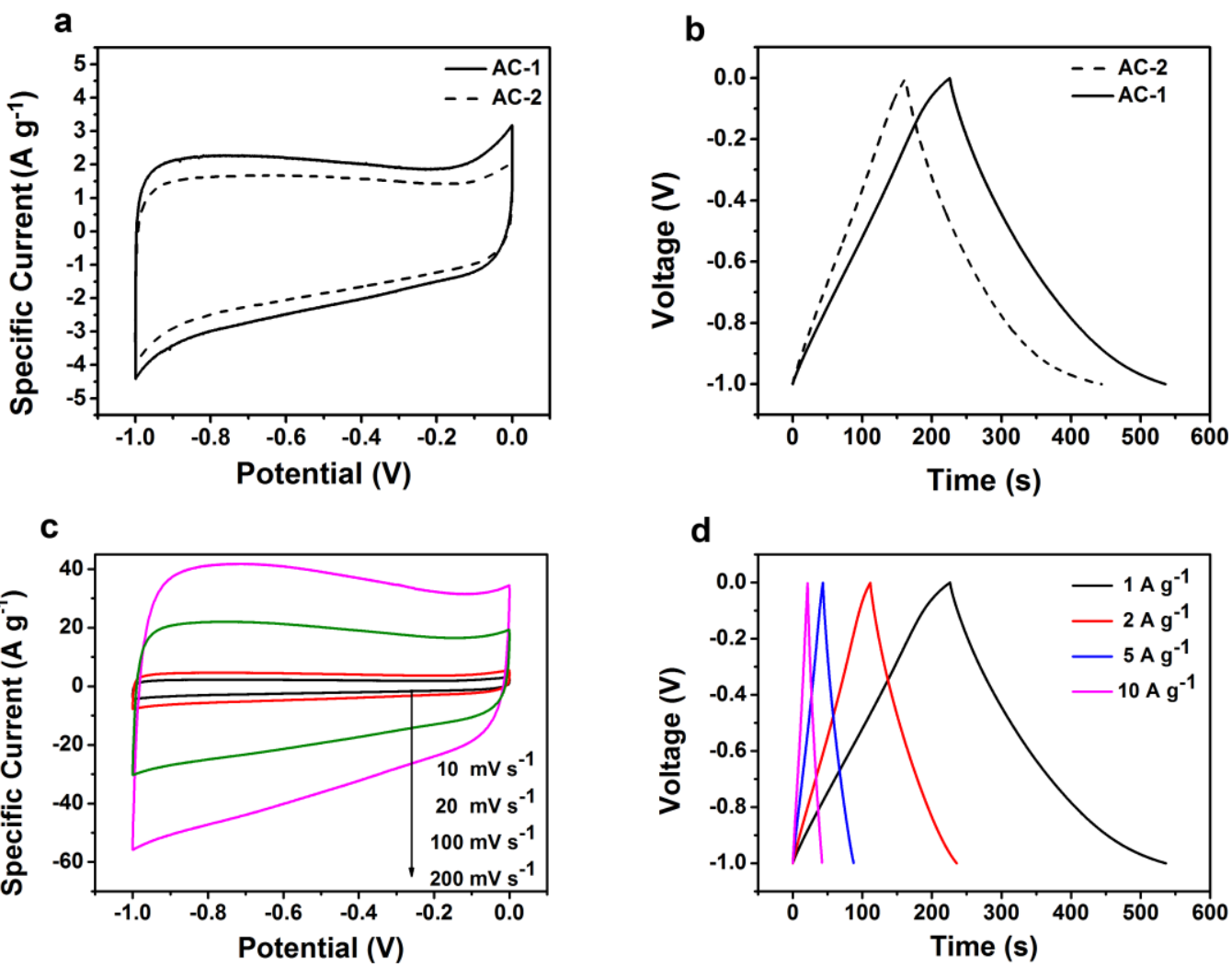

Fig. 4 Three-electrode electrochemical performances in $6 \mathrm{M} \mathrm{KOH}$. (a) CV curves of $\mathrm{AC}-1$ and $\mathrm{AC}-2$ at $10 \mathrm{mV} \mathrm{s}^{-1}$. (b) GCD curves of $\mathrm{AC}-1$ and $\mathrm{AC}-2$ at $1 \mathrm{~A} \mathrm{~g}^{-1}$. (c) $\mathrm{CV}$ curves of AC-1 at different scan rates in $6 \mathrm{M} \mathrm{KOH}$ solution. The arrow indicates the increase of the scan rates from 10 to $200 \mathrm{mV} \mathrm{s}^{-1}$. (d) GCD curves of AC-1 at different specific currents from 1 to $10 \mathrm{Ag}^{-1}$.

Symmetrical two-electrode coin type supercapacitors were constructed using both aqueous $(6 \mathrm{M} \mathrm{KOH})$ and ionic liquids $\left(\mathrm{EMIMBF}_{4}\right)$ as electrolytes. The nearly rectangular CV profiles and the symmetrical triangular charge/discharge plots are well maintained at different scan rates and specific currents within the potential window of $1 \mathrm{~V}$ using $6 \mathrm{M} \mathrm{KOH}$ electrolyte (Fig. 5 and S4), indicating that the EDL mechanism 
dominates the electrochemical behavior of $\mathrm{AC}-1$ for the aqueous supercapacitors. The maximum specific capacitance of the symmetrical AC-1 cell is $57 \mathrm{~F} \mathrm{~g}^{-1}$ with surface density of $0.45 \mathrm{mg} \mathrm{cm}^{-2}$ at $0.25 \mathrm{~A} \mathrm{~g} \mathrm{~g}^{-1}$ according to the discharge curves, corresponding to $228 \mathrm{~F} \mathrm{~g}^{-1}$ for a single electrode, which is consistent with the value in the three-electrode cell. The coulombic and energy efficiencies are $92.7 \%$ and $73.1 \%$ in $\mathrm{KOH}$ electrolyte, respectively. To evaluate the performance for practical application, the AC-1 electrode with surface density of $10 \mathrm{mg} \mathrm{cm}^{-2}$ has been loaded on nickel foams. Even with such a large mass loading, the AC-1 electrode still shows a specific capacitance of $46 \mathrm{~F} \mathrm{~g}^{-1}$ at $0.25 \mathrm{~A} \mathrm{~g}^{-1}$. When EMIMBF$_{4}$ was employed as electrolyte instead of $\mathrm{KOH}$, it is found that the threshold potentials of ionic liquid decomposition and carbon oxidation for AC-1 in the ionic liquid electrolyte are -1.9 and $0.9 \mathrm{~V}$ vs. Pt in a three-electrode cell, giving a stable electrochemical window of 2.8 V, as shown in Fig. S5 [27,28]. The EIS method also has been used to analyze the potential window in the three electrode cell in $\mathrm{EMIMBF}_{4}$. According to Fig S6, the shapes of the Nyquist plots in low frequency obviously deviate from a vertical line when the voltage range exceeds -1.9 to $0.9 \mathrm{~V}$. The phase angles in Fig. S7 for -2 and $1 \mathrm{~V}$ of the initial potentials are both below $-40^{\circ}$, which is far from $-90^{\circ}$ corresponding to an ideal capacitive characteristic [29]. The CV measurements of the AC-1 cell in the $\mathrm{EMIMBF}_{4}$ electrolyte also shows rectangular profiles with potential window of $2.8 \mathrm{~V}$ at different scan rates in Fig. 6a. The highest specific capacitance of the AC-1 cell in the EMIMBF 4 electrolyte is calculated to be $17 \mathrm{~F} \mathrm{~g}^{-1}$ with surface density of $0.45 \mathrm{mg} \mathrm{cm}^{-2}$ and $16 \mathrm{~F} \mathrm{~g}^{-1}$ with surface density of $10 \mathrm{mg} \mathrm{cm}^{-2}$ at $0.25 \mathrm{~A} \mathrm{~g}^{-1}$ by the 
discharge curves in the GCD process (Fig 6b, c and S8), corresponding to a specific capacitance of $68 \mathrm{~F} \mathrm{~g}^{-1}$ for a single electrode. The coulombic and energy efficiencies are $87.8 \%$ and $60.1 \%$ in $\mathrm{EMIMBF}_{4}$ electrolyte, respectively, which are lower than those in $\mathrm{KOH}$, indicating low reversibility wihin $2.8 \mathrm{~V}$ in $\mathrm{EMIMBF}_{4}[30,31]$. The specific energy of AC-1 in the cell is calculated to be $18.5 \mathrm{Wh} \mathrm{kg}^{-1}$ for the cell.
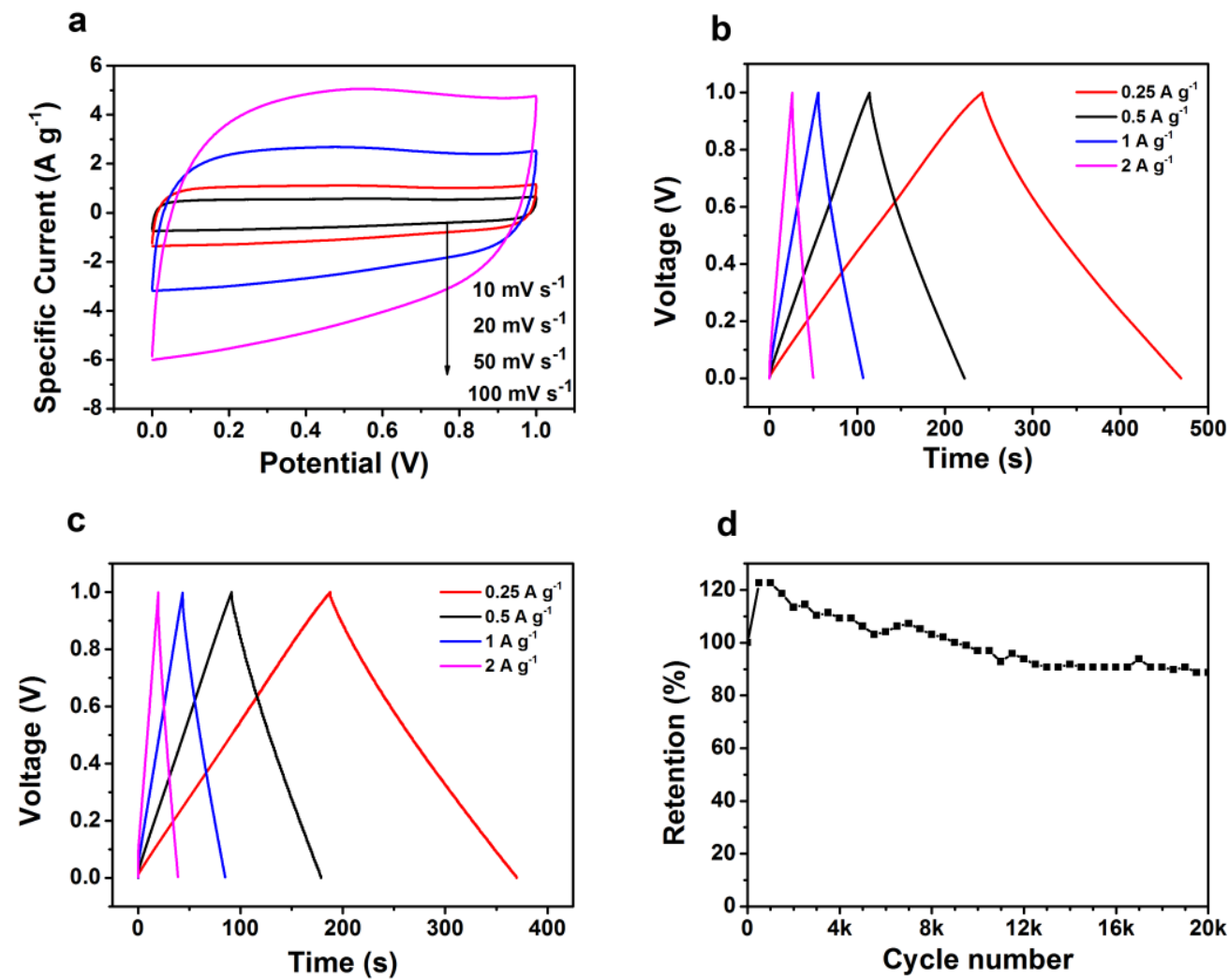

Fig. 5 Two-electrode electrochemical performances of the AC-1 cell in $6 \mathrm{M} \mathrm{KOH}$. (a) $\mathrm{CV}$ curves at different scan rates, the arrow indicates the increase of the scan rates from 10 to $100 \mathrm{mV} \mathrm{s}^{-1}$. (b) GCD curves at different specific currents with the surface density of $0.45 \mathrm{mg} \mathrm{cm}^{-2}$ on the nickel foils and (c) the surface density of $10 \mathrm{mg} \mathrm{cm}^{-2}$ on the nickel foams. (d) Cyclic performance of $\mathrm{AC}-1$ in $\mathrm{KOH}$ at $1 \mathrm{~A} \mathrm{~g}^{-1}$. 
The cyclic performances of $\mathrm{AC}-1$ in $\mathrm{KOH}$ and $\mathrm{EMIMBF}_{4}$ at room temperature are present in Fig. 5d, 6d and S9. 88.6 and $63.6 \%$ of the initial capacitance can be retained for $\mathrm{AC}-1$ in the $6 \mathrm{M} \mathrm{KOH}$ and $\mathrm{EMIMBF}_{4}$ electrolytes at $1 \mathrm{~A} \mathrm{~g}^{-1}$ after the 20000 and 3000 cycles, respectively. Interestingly, the AC-1 cell using $\mathrm{KOH}$ as electrolyte shows a progressive increase in the specific capacitance before 1000 cycles, which is attributed to better wetting at the electrolyte/electrode interface, especially in deep pores, after cycles [2,32-34]. In case of $\mathrm{EMIMBF}_{4}$, after 500 cycles, the AC-1 only maintains $80 \%$ of the initial capacitance. The rapid deterioration and relative narrow potential window in $\mathrm{EMIMBF}_{4}$ are attributed to the irreversible redox reaction arising from 8.79 at\% $\mathrm{C}-\mathrm{O}$ bond in $\mathrm{AC}-1$ [35-37]. However, it is believed that very low oxygen content in carbon also can be realized by the new one-step method.

\section{Conclusions}

Activated carbon was synthesized by a simple one-step calcination of deoxygenated agar in a hot $\mathrm{KOH}$ aqueous solution. The deoxygenation course of soluble agar in the hot $\mathrm{KOH}$ solution omits high temperature carbonization in the conventional technology and leads to molecular level activation of agar in subsequent one-step calcination. The one-step activated carbon (AC-1) has superior specific surface area of $1672 \mathrm{~m}^{2} \mathrm{~g}^{-1}$ and total pore volume of $0.81 \mathrm{~cm}^{3} \mathrm{~g}^{-1}$, obviously higher than those of the activated carbon by the conventional two-step method (AC-2). As a result, the 
maximum specific capacitance of $226 \mathrm{~F} \mathrm{~g}^{-1}$ in the $\mathrm{KOH}$ electrolyte is obtained for $\mathrm{AC}-1$ is higher than $164 \mathrm{~F} \mathrm{~g}^{-1}$ for $\mathrm{AC}-2$. However, $\mathrm{AC}-1$ also shows a rapid deterioration and relative narrow potential window in $\mathrm{EMIMBF}_{4}$ due to 8.79 at $\% \mathrm{C}-\mathrm{O}$ bond in AC-1. This study provides potentially economical and effective means for the production of commercial activated carbon with high porosity for supercapacitors.
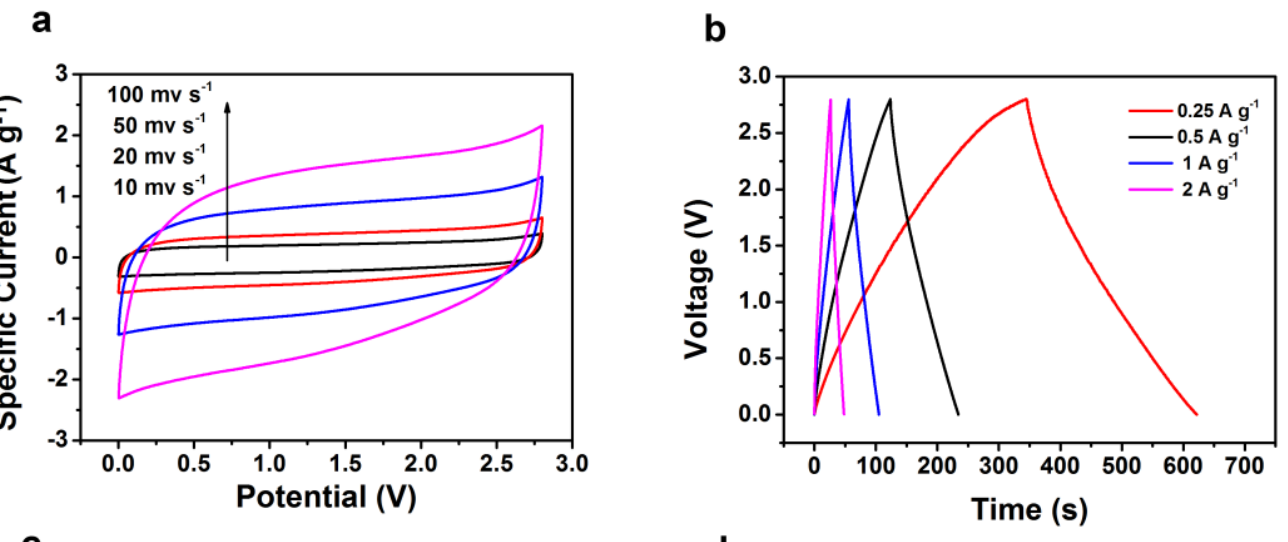

C

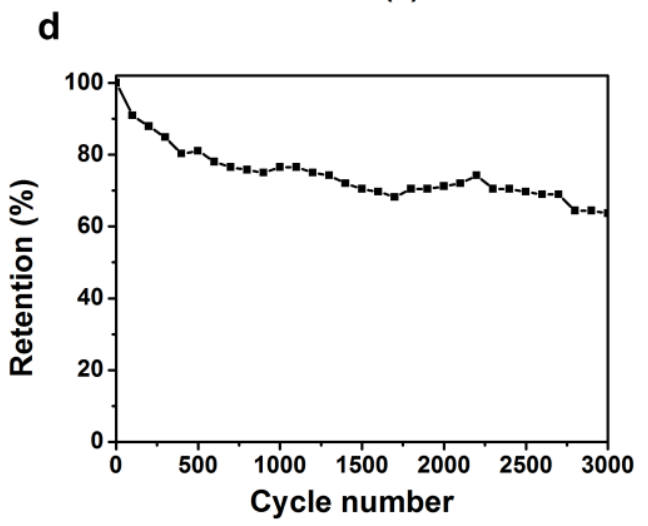

Fig. 6 Two-electrode electrochemical performances of the AC-1 cell in $\mathrm{EMIMBF}_{4}$. (a) $\mathrm{CV}$ curves at different scan rates, the arrow indicates the increase of the scan rates from 10 to $100 \mathrm{mV} \mathrm{s}^{-1}$. (b) GCD curves at different specific currents with the surface density of $0.45 \mathrm{mg} \mathrm{cm}^{-2}$ on the nickel foils and (c) the surface density of $10 \mathrm{mg} \mathrm{cm}^{-2}$ on the nickel foams. (d) Cyclic performance of AC-1 in EMIMBF 4 at $1 \mathrm{~A} \mathrm{~g}^{-1}$. 


\section{Acknowledgement}

This work was partially supported by the financial support from the Department of

Education of Hubei Provincial People's Government (Scientific Research Program, Q20161104). 


\section{References}

[1] Chen GZ. Supercapacitor and supercapattery as emerging electrochemical energy stores. Inter Mater Rev 2017;62:173-202.

[2] González A, Goikolea E, Andoni Barrena J, Mysyk R. Review on supercapacitors: technologies and materials. Renew Sust Energy Rev 2016;58:1189-206

[3] Chen HY, Zeng S, Chen MH, Zhang YY, Li QW. Fabrication and functionalization of carbon nanotube films for high-performance flexible supercapacitors. Carbon 2015;92:271-96.

[4] Gromadskyi DG, Chae JH, Norman SA, Chen GZ. Correlation of energy storage performance of supercapacitor with iso-propanol improved wettability of aqueous electrolyte on activated carbon electrodes of various apparent densities. App Energy 2015;159:39-50.

[5] Zhu YW, Murali S, Stoller MD, Ganesh KJ, Cai WW, Ferreira PJ, et al. Carbon-based supercapacitors produced by activation of graphene. Science 2011;332:1537-40.

[6] Zheng C, Zhou XF, Cao HL, Wang GH, Liu ZP. Synthesis of porous graphene/activated carbon composite with high packing density and large specific surface area for supercapacitor electrode material. J Power Sources 2014;258:290-6.

[7] Gao S, Wang K, Du ZL, Wang YL, Yuan AB, Lu W, et al. High power density electric double-layer capacitor based on a porous multi-walled carbon nanotube 
microsphere as a local electrolyte micro-reservoir. Carbon 2015;92:254-61.

[8] Chen Y, Zhang X, Zhang DC, Yu P, Ma YW. High performance supercapacitors based on reduced graphene oxide in aqueous and ionic liquid electrolytes. Carbon 2010;49:573-80

[9] Wu SL, Chen GX, Kim NY, Zhu YW. Creating pores on graphene platelets by low-temperature $\mathrm{KOH}$ activation for enhanced electrochemical performance. Small 2012;12:2376-84.

[10] Wei XJ, Zou HL, Gao SY. Chemical crosslinking engineered nitrogen-doped carbon aerogels from polyaniline-boric acid-polyvinyl alcohol gels for high performance electrochemical capacitors. Carbon 2017;123:471-80.

[11] Karnan M, Subramani K, Srividhy PK, Sathish M. Electrochemical studies on corncob derived activated porous carbon for supercapacitors application in aqueous and non-aqueous electrolytes. Electrochim Acta 2017;228:586-96.

[12] Tooming T, T. Thomberg, H. Kurig, Janes A, Lust E. High power density supercapacitors based on the carbon dioxide activated D-glucose derived carbon electrodes and 1-ethyl-3-methylimidazolium tetrafluoroborate ionic liquid. J Power Sources 2015;280: 667-77.

[13] Li X, Wei BQ. Supercapacitors based on nanostructured carbon. Nano Energy 2013:2:159-173.

[14] Li YS, Sun IW, Chang JK, Sua CJ. Doped butylmethylpyrrolidinium-dicyanamide ionic liquid as an electrolyte for $\mathrm{MnO}_{2}$ supercapacitors. J Mater Chem 2012;22:6274-9. 
[15] Sun W, Lipka SM, Swartz C, William D, Yang FQ. Hemp-derived activated carbons for supercapacitors. Carbon 2016;103:181-92.

[16] Chae JH, Chen GZ. 1.9 V aqueous carbon-carbon supercapacitors with unequal electrode capacitances. Electrochim Acta 2012;86:248-54.

[17] Zhang DD, Zhao JH, Feng C, Zhang JJ. Scalable synthesis of hierarchical macropore-rich activated carbon microspheres assembled by carbon nanoparticles for high rate performance supercapacitors. J Power Sources $2017 ; 342: 363-70$

[18] Wei L, Sevilla M, Fuertes AB, Mokaya R, Yushin G. Polypyrrole-derived activated carbons for high-performance electrical double-layer capacitors with ionic liquid electrolyte. Adv Func Mater 2012;22:827-34.

[19] Díez N, Mysyk R, Zhang W, Goikolea E, Carriazo D. One-pot synthesis of highly activated carbons from melamine and terephthaldehyde as electrodes for high energy aqueous supercapacitors. J Mater Chem A 2017;5:14619-29.

[20] Tian X, Ma HR, Li Z, Yan SC, Ma L, Yu F, et al. Flute type micropores activated carbon from cotton stalk for high performance supercapacitors. J Power Sources 2017;359: 88-96.

[21] Hwang JY, Li MP, El-Kady MF, Kaner RB. Next-generation activated carbon supercapacitors: a simple step in electrode processing leads to remarkable gains in energy density. Adv Funct Mater 2017;27:1605745.

[22] Rehman S, Gu XX, Khan K, Mahmood N, Hou YL, Huang XX, et al. 3D vertically aligned and interconnected porous carbon nanosheets as sulfur 
immobilizers for high performance lithium-sulfur batteries. Adv Energy Mater 2016;6:1502518.

[23] Balahmar N, Al-Jumialy AS, Mokaya R. Biomass to porous carbon in one step: directly activated biomass for high performance $\mathrm{CO}_{2}$ storage. J Mater Chem A 2017;10:1039.

[24] Laksaci H, Khelifi A, Trari M, Addoun A. Synthesis and characterization of microporous activated carbon from coffee grounds using potassium hydroxides. $\mathrm{J}$ Clean Prod 2017;147:254-62.

[25] Li M, Li W, Liu SX. Hydrothermal synthesis, characterization, and $\mathrm{KOH}$ activation of carbon spheres from glucose. Carbohyd Res 2011;346:999-1004.

[26] Brunauer S, Emmett P, Teller E. Adsorption of gases in multimolecular layers. J Am Chem Soc 1938;60:309.

[27] Li J, Tang J , Yuan JS, Zhang K, Shao QG, Qin LC, et al. Interactions between graphene and ionic liquid electrolyte in supercapacitors. Electrochim Acta 2016;197:64-91.

[28] Lewandowski A, Olejniczak A, Galinski M, Stepniak I. Performance of carbon-carbon supercapacitors based on organic, aqueous and ionic liquid electrolytes. J Power Sources 2010;195:5814-9.

[29] Härmas M, Thomberg T, Romann T, Jänes A, Lust E. Carbon for energy storage derived from granulated white sugar by hydrothermal carbonization and subsequent zinc chloride activation. J Electrochem Soc 2017;164: A1866-72.

[30] Weingarth D, Foelske-Schmitz A, Kötz R. Cycle versus voltage hold - Which is 
the better stability test for electrochemical double layer capacitors. J Power Sources 2013;225:84-8.

[31] Kötz R, Carlen M. Principles and applications of electrochemical capacitors. Electrochim Acta 2000;45:2483-98.

[32] Chen Y, Zhang X, Zhang HT, Sun XZ, Zhang DC, Ma YW. High-performance supercapacitors based on a graphene-activated carbon composite prepared by chemical activation. RSC Adv 2012;2:7747-53.

[33] Hahn M, Barbieri O, Gallay R, Kötz R. A dilatometric study of the voltage limitation of carbonaceous electrodes in aprotic EDLC type electrolytes by charge-induced strain. Carbon 2006;44:2523-33.

[34] Hastak RS, Sivaraman P, Potphode DD, Shashidhara K, Samui AB. All solid supercapacitor based on activated carbon and poly [2,5-benzimidazole] for high temperature application. Electrochim Acta 2012;59:296-303.

[35] Eskusson J, Jänes A, Kikas A, Matisen L, Lust E. Physical and electrochemical characteristics of supercapacitors based on carbide derived carbon electrodes in aqueous electrolytes. J Power Sources 2011;196:4109-16.

[36] Li SM, Yang SY, Wang YS, Tsai HP, Tien HW, Hsiao ST, Liao WH, Chang CL, Ma CCM, Hu CC. N-doped structures and surface functional groups of reduced graphene oxide and their effect on the electrochemical performance of supercapacitor with organic electrolyte. J Power Sources 2015;278:218-29.

[37] Tian WQ, Gao QM, Tan YL, Zhang YL, Xu JD, Li ZY, Yang K, Zhu LH, Liu ZP. Three-dimensional functionalized graphenes with systematical control over the 
interconnected pores and surface functional groups for high energy performance supercapacitors. Carbon 2015;85:351-62. 